\title{
Cognitive Coping as a Mechanism of Change in Cognitive-Behavioral Therapy for Fear of Flying: A Longitudinal Study With 3-Year Follow-Up
}

\author{
Bert Busscher ${ }^{1,2}$ and Philip Spinhoven ${ }^{2,3}$ \\ ${ }^{1}$ VALK Foundation \\ ${ }^{2}$ Leiden University \\ ${ }^{3}$ Leiden University Medical Center
}

Objective: To examine the predictive value of cognitive coping strategies at pretreatment and the value of changes in these strategies during cognitive-behavioral treatment for aviophobia for long-term therapy results. Method: Data from baseline, after therapy at 2 months, short-term follow-up at 5 months, and long-term follow-up at 41 months were analyzed $(N=59)$. Results: Participants were in a long-term process of change, which continued positively after therapy for maladaptive cognitive coping strategies. The use of cognitive coping strategies at baseline was not predictive of long-term outcome. However, a greater increase in the use of adaptive coping strategies, and more importantly, a greater decrease in the use of maladaptive coping strategies were predictive of improvements indicated in selfreport of flight anxiety and actual flight behavior at long-term follow-up. Conclusion: Improvement of maladaptive cognitive coping strategies is possibly a key mechanism of change in cognitive-behavioral therapy for aviophobia. (c) 2016 Wiley Periodicals, Inc. J. Clin. Psychol. 73:1064-1075, 2017.

Keywords: emotion regulation; mechanism of change; aviophobia; anxiety; CBT

Individual differences in emotion regulation are associated with well-being, successful functioning and mental health (Aldao et al., 2010; Berking \& Wupperman 2012; Rooij et al., 2014). The broad concept of emotion regulation refers to the conscious and unconscious physiological, behavioral, and cognitive processes that modulate emotions to respond appropriately to environmental demands (Thompson, 1994). Within emotion regulation, cognitive coping can be seen as a conscious strategy to deal with (primarily) negative events and stressful situations, and the thoughts related to these situations. Some cognitive coping strategies seem more adaptive than others, while the use of certain maladaptive cognitive coping strategies has been linked to psychopathology (Aldao et al., 2010; Rooij et al., 2014; Potthoff et al., 2016). Strikingly, almost no studies of anxiety problems have been done as to whether psychological interventions may change the use of specific coping strategies, and if so, whether these changes predict subsequent reductions in anxiety. The present study examined whether coping strategies at pretreatment and changes in coping strategies during cognitive-behavioral treatment (CBT) are predictive of fear of flying at 3-year follow-up in a group of aviophobic patients.

\section{Coping Styles and Anxiety}

Numerous studies have examined the relationship of different coping styles with anxiety. The preferential and predominant use of cognitive coping strategies can be split into two separate

Bert Busscher and Philip Spinhoven have no financial interests or potential conflicts of interests.

Please address correspondence to: Bert Busscher, Postbox 110, 2300 AC Leiden, The Netherlands. E-mail: psycholoot@home.nl; b.h.busscher@umail.leidenuniv.nl 
styles: an adaptive style inversely associated with anxiety and a maladaptive style positively associated with anxiety. Primarily cross-sectional studies have shown strong and consistent relationships between catastrophizing, rumination, and blaming oneself for what one has experienced, and the reporting of anxiety in all age groups and across groups with different cultural backgrounds. To a lesser extent negative relationships have been found between reappraisal, refocusing on pleasant issues, and thinking how to handle negative events on the one hand and the reporting of anxiety on the other (Garnefski \& Kraaij, 2007; Potthoff et al., 2016).

As the use of a predominantly maladaptive cognitive coping style is more strongly related to psychopathology than is the parsimonious use of an adaptive coping style, it might be more beneficial to focus therapy on improving maladaptive cognitive coping strategies rather than enhancing reappraisal and other adaptive coping strategies (Aldao et al., 2010). For example, Krijn (2005; results partially published in Krijn et al., 2007) reports significant reduction in the use of maladaptive coping strategies from pretreatment to posttreatment in 35 patients after CBT for aviophobia, and no changes in the use of adaptive coping strategies; however, she failed to relate these changes to outcome.

\section{Fear of Flying}

The present study examined within a group of people with aviophobia the relationship between (changes in) coping style and (changes in) anxiety and avoidance behavior during treatment and at 3-year follow-up. It is estimated that more than a third of all people find flying difficult and distressing. Fear of flying (aviophobia) is a debilitating disorder that affects $10 \%-15 \%$ of the general population in the Western world (Oakes \& Bor, 2010a; Ekeberg, Fauske, \& Berg-Hansen, 2014). Nearly all of these people either avoid flying, or fly with the help of medication, drugs, or alcohol.

The preferred treatment method is CBT combined with exposure. The acclaimed success rates of therapy range from $67 \%$ to $96 \%$ (Oakes \& Bor, 2010b). People with aviophobia seeking treatment are found to have a dispositional tendency toward maladaptive strategies, including avoidance behavior, to cope with their anxiety (Kraaij, Garnefski, \& Van Gerwen, 2003). In general, phobic people try to avoid both actual and cognitive confrontation with their feared object (Pittig, Brand, Pawlikowski, \& Alpers, 2014; Sadaat, Izadi, Ahmadi, \& Shahyad, 2014). Cognitive avoidance can be seen as conscious suppression of unwanted thoughts. Such attempts can be counterproductive, leading to increased accessibility of the suppressed thoughts; this then results in hypersensitivity to anxiety-related thoughts and symptoms (Wenzlaff \& Wegner, 2000). For the present study, we based our analyses on the aggregated adaptive and maladaptive coping styles mentioned above. We included cognitive avoidance among the maladaptive cognitive coping styles.

\section{Hypotheses}

Our first study hypothesis was (a) that participants with aviophobia who use maladaptive strategies more often than adaptive strategies at the start of treatment will profit less from treatment. While cognitive coping strategies refer to personal coping styles, these styles are not rigid and therefore amenable to change by targeted intervention (Garnefski \& Kraaij, 2007). So, we further expected (b) that CBT would result in an enhanced use of adaptive coping strategies, and especially a reduced use of maladaptive coping strategies. Finally, we hypothesized (c) that in particular a reduction of the maladaptive coping style during treatment would predict a better long-term therapy outcome.

\section{Methods}

\section{Participants}

The sample in this study comprised 59 adults ( $28 \mathrm{men}$ ), with an average age of 42.3 years (standard deviation $[S D]=11.0$, range 20-61). The present study was part of a longitudinal 
study on psychophysiological aspects associated with fear of flying (Busscher, Spinhoven, \& de Geus, 2015). The original study started with 127 participants who had applied for therapy to overcome their aviophobia; 17 participants did not complete therapy. Study dropout from the original study was considerable over the course of time $(n=31)$ and, once excluded from the original study, study dropouts were no longer required to fill out the Cognitive Emotion Regulation Questionnaire-Flight (CERQ-F). Consequently, 79 participants provided CERQ-F data at the end of treatment (T1). Inclusion criteria for the present study were complete data on the CERQ-F at intake (T0), end of treatment (T1) and at 5-month follow-up (T2). Only 59 of the 79 participants attended this optional short-term follow-up (T2). Therefore, another 20 participants were lost for analysis. All 59 completers provided long-term outcome data at T3 (3 years after short-term follow-up).

\section{Materials}

Visual Analogue Flight Anxiety Scale (VAFAS) (Nousi et al., 2008). This singleitem one-tailed scale was used to examine to what extent participants were anxious about flying. The scale ranges from 0 (no flight anxiety) to 10 (terrified or extreme flight anxiety).

CERQ-F. The CERQ-F is an adaptation of the 36-item CERQ (Kraaij, Garnefski, \& Gerwen, 2003) to measure cognitive coping with flying specifically and was extended by the originators with an additional tenth subscale to assess the use of cognitive avoidance strategies.

This 40-item self-report inventory assesses the following 10 conceptually different cognitive coping strategies: (a) Self-blame, thoughts of blaming yourself for what you have experienced; (b) Acceptance, thoughts of accepting what you have experienced and resigning yourself to what has happened; (c) Rumination, thinking about the feelings and thoughts associated with the negative event; (d) Refocus Positive, thinking about joyful and pleasant issues instead of thinking about the actual event; (e) Refocus Planning, thinking about what steps to take and how to handle the negative event; (f) Positive Reappraisal, thoughts attaching a positive meaning to the event in terms of personal growth; (g) Putting into Perspective, thoughts that play down the seriousness of the event or emphasize its relativity when compared to other events; (h) Catastrophizing, thoughts explicitly emphasizing the terror of an experience; (i) Other-blame, thoughts that put on others the blame for what you have experienced, and (j) Cognitive Avoidance, thinking about how to avoid thoughts of the negative event.

All 40 items are rated on a 5-point Likert-type scale ranging from 1 (almost never) to 5 (almost always). The score of a subscale is obtained by adding up the scores of the four items belonging to the particular subscale, and it ranges from 4 (never used) to 20 (often used cognitive coping strategy).

The original 36-item CERQ has been used in the study of a wide variety of domains, including depression, anxiety, and mental health. Construct validity, reliability, and test-retest reliability of the CERQ proved to be good within subgroups of adolescents, adults, elderly people, and psychiatric patients. The inventory has been translated into many languages and shows good cross-cultural consistency. The test-retest correlations of the CERQ indicate that cognitive coping strategies are relatively stable over time, although not as stable as personality traits (Garnefski \& Kraaij, 2007; Potthoff et al., 2016).

Within the CERQ subscales exists a second-order hierarchical structure with two composite subscales (Potthoff et al., 2016). The second-order adaptive coping style scale is the simple straight sum of all 12 items from the subscales of Refocus-Positive, Refocus-Planning, and Positive-Reappraisal and ranges from 12 to 60 . The 16-item maladaptive coping style scale is the sum score of all items belonging to the subscales of Self-Blame, Rumination, Catastrophizing, and Avoidance and ranges from 16 to 80 . The internal consistency of the adaptive and maladaptive scales in the present study was good, with Cronbach's alpha ranging from .85 to .89 for the adaptive scale and from .85 to .88 for the maladaptive scale. For clarity, we use the term coping strategies when referring to the 10 different subscales of the CERQ-F, and coping styles when referring to the two second-order composite subscales. 


\section{Treatment}

Participants in this study followed a highly standardized treatment program for fear of flying at a specialized institution in The Netherlands. The program started with a diagnostic assessment followed by up to four individual 1-hour CBT sessions. Then participants joined a 2-day cognitive-behavioral group treatment (CBGT), lasting 20 hours in total. A group comprised an average of eight participants. The second day of CBGT was focused on exposure and included a guided return flight on a commercial airliner. Information on cognitive emotion regulation and training in coping skills were included in the individual CBT and the group CBGT. Three months after CBGT, participants were invited to attend an optional single 3-hour follow-up session. Details of the therapeutic protocol have been published elsewhere (Van Gerwen, Spinhoven, \& Van Dyck, 2006).

\section{Data Collection Procedure}

Coping strategies (CERQ-F) and flight anxiety (VAFAS) were assessed concurrently at three moments in time. Baseline measurements (T0) were integrated into the existing intake before start of treatment. The second assessment (T1) was performed 2 months after baseline, at the end of CBGT. At 5 months after baseline, a short-term follow-up assessment (T2) took place during the optional 3-hour follow-up session. Three years after short-term follow-up, participants furnished long-term effects of therapy (T3) by providing a flight anxiety score (VAFAS) and reporting their number of flights flown within these 3 years. E-mail was used to collect this long-term data. CERQ-F data was not collected at T3.

\section{Statistical Analysis}

Chi-square tests, independent t-tests and one-way analyses of variance were used to examine baseline (T0) and end of treatment (T1) differences in flight anxiety and coping strategies between completers (i.e. participants with a CERQ-F score at T2) and dropouts (those who did not furnish a CERQ-F score at T2). Treatment effects on VAFAS were assessed with paired samples $t$ tests. Changes in coping strategies and coping styles between T0, T1, and T2 were assessed with paired samples $t$ tests.

Multiple regression analyses (MRA) were used to assess the relationship between cognitive coping and long-term treatment effect (T3). Long-term treatment effect was operationalized as the flight anxiety scores 3 years after short-term follow-up (VAFAS), and number of flights taken in this 3-year period. Number of flights was first log transformed to obtain a normal distribution. Predictor variables were the scores on the adaptive and maladaptive scales of the CERQ-F at T0, and residualized changes on these scales from baseline to short-term followup (T0-T2). Theoretically, merging separate indicators into composite measures extends the range of scores available and increases power in regression analyses by reducing the number of predictor variables.

Preliminary analyses revealed that the use of specific coping strategies continued to change after therapy (T1) up to short-term follow-up (T2). We therefore used the T2 values instead of the T1 values in the MRA on changes in coping style, because these short-term followup coping scores best reflected the coping styles used by participants during the 3 years after therapy ${ }^{1}$. Because anxiety severity and cognitive coping are known to be strongly interrelated, we performed all our analyses with corresponding anxiety scores as covariates in the first step of the analyses. Cognitive coping scores were added in a second step to assess whether they significantly predicted outcome over and above anxiety severity at T0 or changes in anxiety severity between $\mathrm{T} 0$ and $\mathrm{T} 2$ when appropriate.

Several analyses were performed. To test whether coping styles at baseline were predictive of long-term treatment results, flight anxiety at baseline (VAFAS T0) was entered in the first step

\footnotetext{
${ }^{1}$ Analyses with $\mathrm{T} 1$ values produced similar results.
} 
of the MRA. The baseline (T0) scores of the adaptive and maladaptive scales were added in a second step. Second, we tested whether changes in coping style during treatment until shortterm follow-up (T0-T2) were predictive of changes in flight anxiety from short-term follow-up to 3 years later (T2-T3) and in number of flights flown in that period. We calculated residualized change scores and used these throughout the analyses.

Changes in flight anxiety during treatment until short-term follow-up (VAFAS T0-VAFAS T2) were entered in the first step of the MRA; changes in adaptive and maladaptive coping styles during treatment until short-term follow-up (T0-T2) were entered in the second step. By doing so, results from the second step of the MRA indicate the association of changes in coping styles from baseline to short-term follow-up with maintenance of treatment outcome, over and above changes in flight anxiety from baseline to short-term follow-up.

\section{Results}

\section{Dropout Analyses}

Statistical analyses showed no significant differences at baseline on demographic and clinical characteristics between treatment dropouts $(\mathrm{n}=17)$, study dropouts $(\mathrm{n}=51)$, and completers $(\mathrm{n}=59)$. Moreover, there were no systematic differences in flight anxiety scores and CERQ-F scores at baseline (T0) as well as in flight anxiety and CERQ-F scores at T1 (end of treatment) between participants who provided CERQ-F data at T2 (short-term follow-up) and participants who did not do so (data not presented, but are available on request).

\section{Clinical Characteristics}

Sociodemographic characteristics of the participants were in line with data previously published on patients applying for therapy at this treatment facility (Busscher, van Gerwen, Spinhoven, $\&$ de Geus, 2010). Only 7\% of the participants had never flown before start of treatment. Of the participants who had flown before, $32 \%$ had flown within 12 months of their application for therapy. The majority, however, had not flown for more than 2 years, while $29 \%$ had not flown for more than 10 years. The mean time between application for therapy and the last flight was 5.1 years $(S D=8.1)$. Figure 1 depicts flight anxiety scores at baseline $(\mathrm{T} 0)$, end of treatment (T1), short-term follow-up (T2), and 3 years after short-term follow-up (T3). Cohen's d showed large effect sizes for the reduction in flight anxiety between baseline and all other measurement moments, ranging from 4.0 at short-term follow-up to 2.6 at long-term follow-up.

\section{CERQ-F Strategies}

Table 1 shows values for the 10 subscales of the CERQ-F at the different assessment moments. Significant changes were seen over time in adaptive and maladaptive strategies. The maladaptive coping strategies Self-Blame, Rumination, Catastrophizing, and Avoidance were used significantly less often at the end of treatment (T1) and short-term follow-up (T2) than at baseline (T0). The adaptive coping strategies Refocus-Positive and Positive-Reappraisal were used significantly more often at $\mathrm{T} 1$ and $\mathrm{T} 2$ than at $\mathrm{T} 0$. Refocus planning as coping strategy was used significantly more often at T1 than at T0, but the $t$ test between baseline (T0) and short-term follow-up (T2) was not significant. Effect size statistics showed large effect for 6 out of 10 strategies, with catastrophizing and avoidance paramount, Cohen's d 1.61 and 1.71 respectively. Participants showed clinically significant improvement in cognitive coping strategies, from pretreatment scores matching those of dysfunctional populations to posttreatment responses matching those of a normal population. 


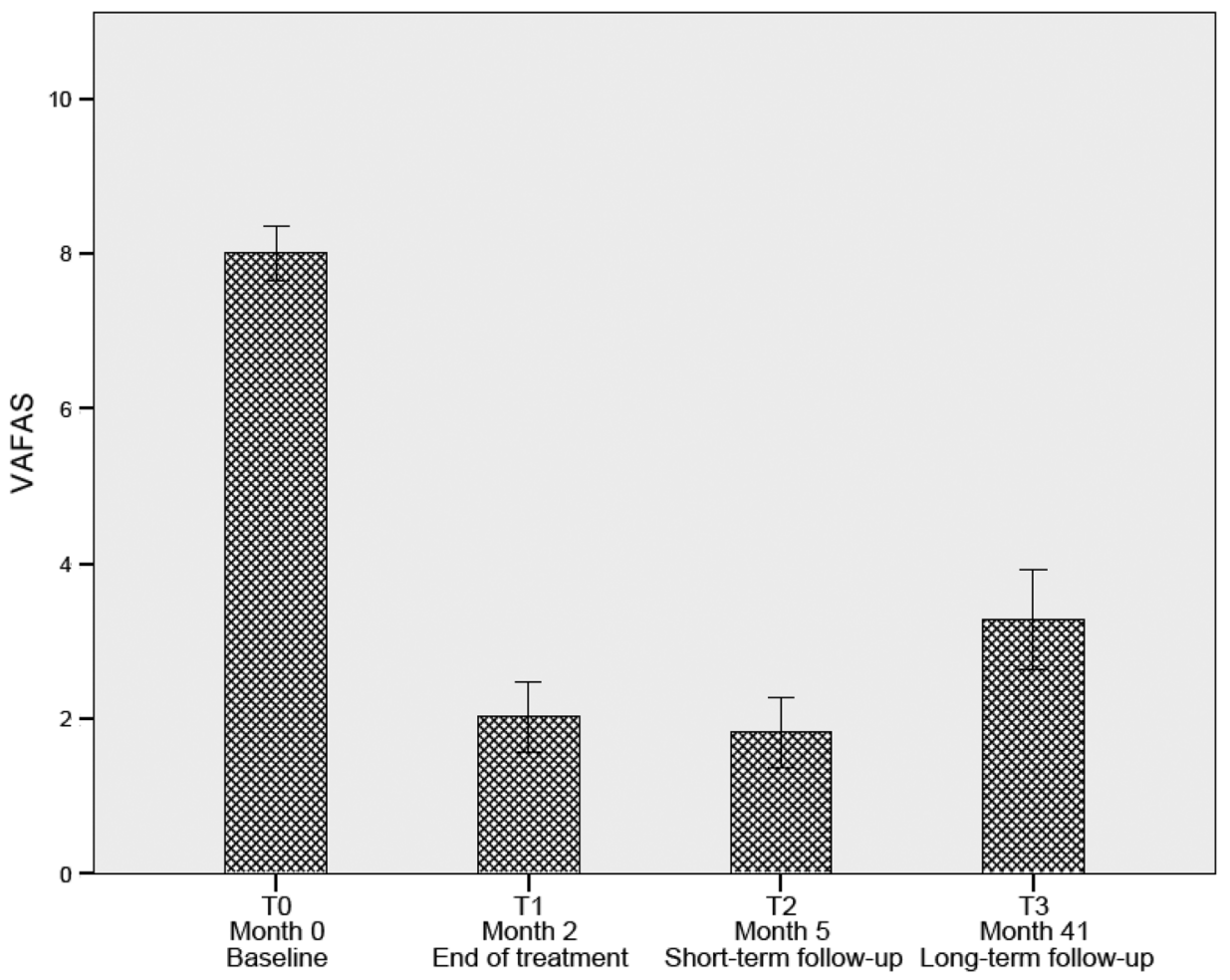

Figure 1. Bar chart of flight anxiety scores (VAFAS) at baseline (T0), end of treatment (T1), short-term follow-up (T2), and long-term follow-up (T3).

Note. Error Bars reflect 95\% CI. VAFAS = Visual Analogue Flight Anxiety Scale

\section{Adaptive and Maladaptive Coping Styles}

Table 1 also shows values for the adaptive and maladaptive styles at the different assessment moments. Using paired $t$ tests we found a significant persistent decline in the use of the maladaptive coping style from baseline to $\mathrm{T} 1$ and $\mathrm{T} 2$. In contrast, for the adaptive scale we found a significant increase from baseline to T1, followed by a significant decrease from T1 to T2. Still, on the adaptive scale participants had a significantly higher score at T2 than at T0. Cohen's d indicated large changes on both scales, with greater changes on the maladaptive than on the adaptive scale.

\section{Prediction of Outcome by Cognitive Coping at Baseline}

Multiple regression analyses were performed to examine whether long-term therapy outcome (T3) could be predicted by coping styles at baseline (T0). The adaptive and maladaptive coping styles at the start of therapy showed no significant relationship with long-term treatment outcome (Tables 2 and 3).

\section{Long-Term Maintenance of Flight Anxiety by Changes in Coping Style During Treatment}

Changes in coping style during treatment until short-term follow-up (T0-T2) were predictive of changes in flight anxiety from short-term follow-up to 3 years later (T2-T3). The additional explained variance in flight anxiety by changes in adaptive and maladaptive coping style over and above changes in flight anxiety (T0-T2) during treatment was $21 \%$. As shown in Table 2, 


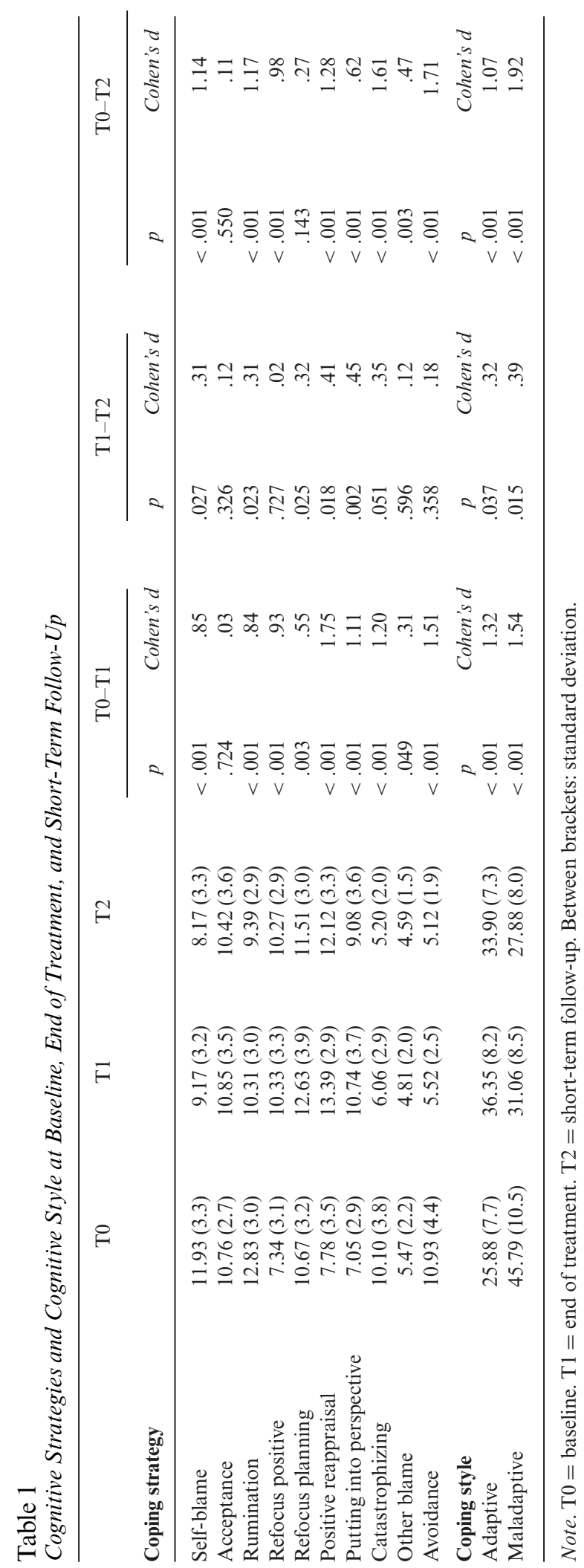


Table 2

Prediction of Long-Term Therapy Outcome (Flight Anxiety; VAFAS at T3) by Adaptive and Maladaptive Coping Styles at Baseline (T0), and Prediction of Long-Term Maintenance of Flight Anxiety (VAFAS T2 - VAFAS T3) by Changes in Adaptive and Maladaptive Coping Styles From Baseline (T0) to Short-Term Follow-Up (T2)

\begin{tabular}{|c|c|c|c|c|c|c|c|}
\hline & Beta & $S E$ & $t$ & $p$ & $\begin{array}{l}\text { Zero-order } \\
\text { correlation }\end{array}$ & $R$ & R-square \\
\hline Flight anxiety at $\mathrm{T} 3$ & \multicolumn{7}{|c|}{ Baseline (T0) } \\
\hline Step 1 & & & & & & .007 & .000 \\
\hline Flight anxiety (T0) & .007 & .275 & .051 & .960 & .007 & & \\
\hline Step 2 & & & & & & .181 & .033 \\
\hline Flight anxiety (T0) & -.009 & .277 & -.063 & .950 & .007 & & \\
\hline Adaptive coping (T0) & -.039 & .043 & -.269 & .789 & -.047 & & \\
\hline Maladaptive coping (T0) & .176 & .034 & 1.19 & .240 & .176 & & \\
\hline $\begin{array}{l}\text { Maintenance of flight } \\
\text { anxiety (T2- T3) }\end{array}$ & \multicolumn{7}{|c|}{ Changes in coping styles (T0-T2) } \\
\hline Step 1 & & & & & & .017 & .000 \\
\hline Flight anxiety (T0-T2) & .017 & .150 & .113 & .910 & .017 & & \\
\hline Step 2 & & & & & & .461 & .214 \\
\hline Flight anxiety (T0-T2) & -.213 & .155 & -1.38 & .174 & .017 & & \\
\hline Adaptive (T0-T2) & .037 & .130 & .261 & .795 & .177 & & \\
\hline Maladaptive (T0-T2) & .505 & .151 & 3.16 & .003 & .418 & & \\
\hline
\end{tabular}

Note. $\mathrm{SE}=$ standard error. $\mathrm{T} 0=$ baseline. $\mathrm{T} 2=$ short-term follow-up. $\mathrm{T} 3=3$ years after short-term follow-up.

Table 3

Prediction of Long-Term Therapy Outcome (Number of Flights) by Adaptive and Maladaptive Coping Styles at Baseline (TO) and by Changes in Adaptive and Maladaptive Coping Styles From Baseline to Short-Term Follow-Up (T2)

\begin{tabular}{|c|c|c|c|c|c|c|c|}
\hline & Beta & $S E$ & $t$ & $p$ & $\begin{array}{l}\text { Zero-order } \\
\text { correlation }\end{array}$ & $R$ & R-square \\
\hline Number of flights & \multicolumn{7}{|c|}{ Baseline (T0) } \\
\hline Step 1 & & & & & & .120 & .014 \\
\hline Flight anxiety (T0) & -.120 & .089 & -.831 & .410 & -.120 & & \\
\hline Step 2 & & & & & & .181 & .033 \\
\hline Flight anxiety (T0) & -.122 & .092 & -.808 & .423 & -.120 & & \\
\hline Adaptive coping (T0) & -.133 & .016 & -.906 & .370 & -.137 & & \\
\hline Maladaptive coping (T0) & .020 & .012 & .132 & .895 & -.001 & & \\
\hline Number of flights & \multicolumn{7}{|c|}{ Changes in coping styles (T0-T2) } \\
\hline Step 1 & & & & & & .275 & .076 \\
\hline Flight anxiety (T0-T2) & -.275 & .127 & -1.94 & .058 & -.275 & & \\
\hline Step 2 & & & & & & .439 & .193 \\
\hline Flight anxiety (T0-T2) & -.117 & .139 & -.759 & .452 & -.275 & & \\
\hline Adaptive (T0-T2) & .290 & .116 & 2.03 & .048 & .206 & & \\
\hline Maladaptive (T0-T2) & -.329 & .136 & -2.05 & .046 & -.306 & & \\
\hline
\end{tabular}

Note. $\mathrm{SE}=$ standard error. $\mathrm{T} 0=$ baseline. $\mathrm{T} 2=3$-month follow-up. $\mathrm{T} 3=3$ years after short-term follow-up. 
this effect was brought about predominantly by changes in maladaptive style. Participants with a greater decrease in the use of the maladaptive coping style reported less increase in flight anxiety from short-term follow-up to 3 years later than did participants who had less decrease in the maladaptive coping style.

\section{Prediction of Number of Flights by Changes in Coping Style During Treatment}

In the 3 years after therapy (T3), participants flew on average 12.4 flights (SD 12.1). Only two participants did not fly, one participant reported 70 flights in this 3-year period. As shown in Table 3, the additional explained variance of flights taken by changes in adaptive and maladaptive coping style over changes in flight anxiety was $12 \%$. Participants with a greater increase in the use of the adaptive coping style reported more flights than did participants with less increase in the use of the adaptive coping style. Participants with a greater decrease in the use of the maladaptive coping style flew more often than did participants who had less decrease in the maladaptive coping style during therapy.

\section{Discussion}

The present study examined, within a relatively large clinical sample of people with aviophobia, the predictive value of using cognitive coping strategies at pretreatment and of changes in these strategies during treatment for long-term therapy results. Results indicated that participants were in a long-term process of change, which in the case of maladaptive cognitive coping strategies continued positively after therapy; this was not the case for adaptive strategies. The use of cognitive coping strategies at baseline was not predictive of therapy outcome. However, a greater increase in the use of adaptive coping strategies and, more importantly, a greater decrease in the use of maladaptive coping strategies during therapy were indicative for less long-term relapse of flight anxiety and more flights flown. The most important significant predictor of maintenance of treatment gains, therefore, was reduced use of a maladaptive coping style over therapy.

In general, within anxiety pathologies the use of the maladaptive coping style seems to play a more prominent role in cognitive emotion regulation than does the adaptive coping style (Garnefski \& Kraaij, 2007; Aldao et al., 2010; Omran, 2011; Rooij et al., 2014). Our findings reinforce the idea that the preponderant presence of a maladaptive coping style might be more detrimental than the relative absence of an adaptive coping style, and that improving maladaptive emotional cognitions during therapy might be more beneficial than improving reappraisal and other adaptive coping strategies.

\section{Maladaptive Strategies Predominate Over Adaptive Strategies}

The beneficial use of generally adaptive strategies might be more context dependent and seem to work less well in situations perceived as uncontrollable, as when traveling in an airplane (Cruess et al., 2002; Aldao et al., 2010). For example, when strapped in a seat during a turbulent flight it might be difficult to attach a positive meaning to the event in terms of personal growth (reappraise), or think about joyful and pleasant issues instead of the actual event (refocus positive). Instead, cognitive avoidance, emphasizing the terror of an experience (catastrophizing) and thinking about the feelings and thoughts associated with the negative event (rumination) might even seem perfectly appropriate in this uncontrollable and frightening situation. The lack of control seems to favor the predominant use of maladaptive strategies.

Furthermore, the use of one maladaptive strategy to deal with a negative event evokes the use of other maladaptive strategies. Rumination without reappraisal will easily lead to catastrophizing and avoidance. This self-reinforcing process favoring maladaptive strategies might override attempts to use more adaptive strategies. 


\section{Implications}

Because maladaptive cognitive coping and emotion regulation strategies can be seen as transdiagnostic factors associated with various psychopathologies such as anxiety, depression and eating disorders, interventions focusing on these factors may have positive effects across a range of disorders (Aldao et al., 2010). A myriad of techniques is available (Mennin, Ellard, Fresco, \& Gross, 2013). Most CBTs are heterogeneous and make use of multiple traditional and newer components such as cognitive reframing, behavioral exposure, behavioral activation, decentering, attention training, and acceptance and tolerance. These therapies can be seen as a blunt instrument that by its heterogeneous nature effectively targets maladaptive coping strategies without concern as to which precise ingredient causes which outcome (Dobson, 2013; Hofmann, Asmundson, \& Beck, 2013; Mennin et al., 2013).

Also in the present study it remains unclear to what extent cognitive interventions such as psychoeducation or in vitro exposure or behavioral interventions such as exposure in the form of a guided flight contributed to reductions in catastrophizing, cognitive avoidance, rumination, and self-blame. Delineating and empirically evaluating treatment components that critically affect the use of certain maladaptive cognitive coping strategies could help to make treatment programs more parsimonious and effective. More effectively targeting specific maladaptive cognitive coping strategies would be greatly enhanced by a more refined knowledge of the functional relationships between certain therapy ingredients and maladaptive coping strategies.

\section{Strengths and Limitations}

The strength of the present study is its use, within a clinical sample of people with aviophobia, of two entirely different clinically relevant long-term outcome measures: a self-report measure indicating flight anxiety and a behavioral measure indicating flight behavior.

Another positive point of the study is its longitudinal design with multiple concurrent assessment moments of coping strategies and flight anxiety. Coping strategies were not, however, assessed at long-term follow-up, thus precluding a cross-lagged panel analysis and formal mediation analysis. Although we could not establish that changes in cognitive coping preceded changes in flight anxiety during active treatment, we did establish that changes in cognitive coping during treatment were predictive for long-term treatment outcome, over and above changes in flight anxiety during treatment. To our knowledge, this is the first study to report the predictive values of changes in maladaptive coping strategies during treatment for subsequent clinical change within the anxiety domain. These results warrant future controlled studies with repeated measurements of anxiety and cognitive coping to formally test the mediating role of changes in cognitive coping.

We also must acknowledge some study limitations. The present study was conducted at a specialized treatment facility, not a research facility. Participants were highly anxious aviophobics who applied for treatment and paid for their treatment. This setting limited research and assessment options considerably. Consequently, follow-up assessment was restricted to flight anxiety and flight behavior.

The present study did not include a control condition, but the considerable changes in flight anxiety and cognitive coping during treatment make it unlikely that these changes in a sample with protracted complaints are the results of passage of time or repeated testing. Participants in the present study followed CBT with cognitive therapy preceding behavioral components, including two exposure flights. Although appealing from a theoretical perspective, the congruent changes in cognitive coping strategies and flight anxiety during treatment do not necessarily imply a causal relationship. Other mechanisms of change, like exposure, could have led to anxiety reductions and consecutive changes in the use of coping strategies. Recently, it has been proposed that consolidation of treatment progress could benefit from postexposure cognitive interventions instead of preexposure cognitive therapy (Craske, Treanor, Conway, Zbozinek, \& Vervliet, 2014; Busscher et al., 2015). Randomized controlled trials are needed to test this assumption. 
Another study limitation is that flight behavior is not only contingent on fear of flying but also influenced by financial aspects as well as work, family, and peer pressure. True as this may be, the clinically important aspect here is that participants, who did not fly in years before therapy, flew on average 12 flights in the 3 years after therapy. Finally, study attrition may limit generalizability of study findings, although there were no significant differences in baseline characteristics between completers and dropouts.

\section{Conclusion}

Diminishing the use of self-blame, rumination, catastrophizing, and avoidance as coping strategies might be a key mechanism of change for people with aviophobia. Multiple coping strategies are used simultaneously, and a cognitive coping strategy that is appropriate in one situation might be less useful in another situation (Doron, Thomas-Ollivier, Vachon, \& Fortes-Bourbousson, 2013). The context of the situation may dictate which coping strategies could best be used, while a flexible use of several strategies dependent on the context could be most useful. The beneficial use of generally adaptive strategies seems to work less well in situations that are perceived as uncontrollable, such as traveling in an airplane (Cruess et al., 2002; Aldao et al., 2010). Cognitive interventions for aviophobia should therefore primarily target the maladaptive coping strategies.

\section{References}

Aldao, A., Nolen-Hoeksema, S., \& Schweizer, S. (2010). Emotion-regulation strategies across psychopathology: A meta-analytic review. Clinical Psychology Review, 30, 217-237.

Berking, M., \& Wupperman, P. (2012). Emotion regulation and mental health: recent Findings, current challenges, and future directions. Current Opinion Psychiatry, 25, 128-134.

Busscher, B., Spinhoven, P., \& de Geus, E. J. C. (2015). Psychological distress and physiological reactivity during in vivo exposure in people with aviophobia. Psychosomatic Medicine, 77, 762-774.

Busscher, B., van Gerwen, L. J., Spinhoven, P., \& de Geus, E. J. C. (2010). Physiological reactivity to phobic stimuli in people with fear of flying. Journal of Psychosomatic Research, 69, 309-317.

Craske, M. G., Treanor, M., Conway, C. C., Zbozinek, T., \& Vervliet, B. (2014). Maximizing exposure therapy: An inhibitory learning approach. Behavior Research and Therapy, 58, 10-23.

Cruess, S., Antoni, M., Hayes, A., Penedo, F., Ironson, G., Fletcher, M., ... Schneiderman, N. (2002). Changes in mood and depressive symptoms and related change processes during cognitive, behavioral stress management in HIV-infected men. Cognitive Therapy and Research, 26, 373-392.

Dobson, K. S. (2013). The science of CBT: Toward a metacognitive model of change. Behavior Therapy, 44, 224-227.

Doron, J., Thomas-Ollivier, V., Vachon, H., \& Fortes-Bourbousson, M. (2013). Relationships between cognitive coping, self-esteem, anxiety and depression: A cluster-analysis approach. Personality and Individual Differences, 55, 515-520.

Ekeberg, O., Fauske, B., \& Berg-Hansen, B. (2014). Norwegian airline passengers are not more afraid of flying after the terror act of September 11. The flight anxiety, however, is significantly attributed to acts of terrorism. Scandinavian Journal of Psychology, 55, 464 468.

Garnefski, N., \& Kraaij, V. (2007). The Cognitive Emotion Regulation Questionnaire - Psychometric features and prospective relationships with depression and anxiety in adults. European Journal of Psychological Assessment, 23, 141-149.

Hofmann, S. G., Asmundson, G. J. G., \& Beck, A. T. (2013). The science of cognitive therapy. Behavior Therapy, 44, 199-212.

Kraaij, V., Garnefski, N., \& Van Gerwen, L. (2003). Cognitive coping and anxiety symptoms among people who seek help for fear of flying. Aviation Space and Environmental Medicine, 74, 273-277.

Krijn, M. (2005). Virtual reality and specific phobias (unpublished dissertation, pp. 1-143). Universiteit van Amsterdam.

Krijn, M., Emmelkamp, P. M. G., Olafsson, R. P., Bouwman, M., Van Gerwen, L. J., Spinhoven, P., .. . Van der Mast, C. A. P. G. (2007). Fear of flying treatment methods: Virtual reality exposure vs. cognitive behavioral therapy. Aviation Space and Environmental Medicine, 78, 121-128. 
Mennin, D. S., Ellard, K. K., Fresco, D. M., \& Gross, J. J. (2013). United we stand: Emphasizing commonalities across cognitive-behavioral therapies. Behavioral Therapy, 44, 234-248.

Nousi, A., Van Gerwen, L., \& Spinhoven, P. (2008). The Flight Anxiety Situations Questionnaire and the Flight Anxiety Modality Questionnaire: Norms for people with fear of flying. Travel Medicine and Infectious Disease, 6(5), 305-310.

Oakes, M., \& Bor, R. (2010a). The psychology of fear of flying (part I): A critical evaluation of current perspectives on the nature, prevalence and etiology of fear of flying. Travel Medicine and Infectious Disease 8, 327-338.

Oakes, M., \& Bor, R. (2010b). The psychology of fear of flying (part II): A critical evaluation of current perspectives on approaches to treatment. Travel Medicine and Infectious Disease, 8, 339-363.

Omran, M. P. (2011). Relationships between cognitive emotion, regulation strategies with depression and anxiety. Open Journal of Psychiatry, 1, 106.

Pittig, A., Brand, M., Pawlikowski, M., \& Alpers, G. W. (2014). The cost of fear: Avoidant decision making in a spider gambling task. Journal of Anxiety Disorders, 28, 326-334.

Potthoff, S., Garnefski, N., Miklosi, M., Ubbiali, A., Dominguez-Sanchez, F. J., Martins, E. C., ... Kraaij, V. (2016). Cognitive emotion regulation and psychopathology across cultures: A comparison between six European countries. Personality and Individual Differences, 98, 218-224.

Rooij, A., Boer, M., Leeden, M., Roorda, L. D., Steultjens, M. P. M., \& Dekker, J. (2014). Cognitive mechanisms of change in multidisciplinary treatment of patients with chronic widespread pain: A prospective cohort study. Journal of Rehabilitation Medicine, 46, 173-180.

Sadaat, S. H., Izadi, M., Ahmadi, K., \& Shahyad, S. (2014). Non-pharmacological treatments for fear of flying. International Journal of Travel Medicine and Global Health, 2, 31-37.

Thompson, R. A. (1994). Emotion regulation: A theme in search of definition. Monographs of the Society for Research in Child Development, 59, 25-52.

Van Gerwen, L. J., Spinhoven, P., \& Van Dyck, R. (2006). Behavioral and cognitive group treatment for fear of flying: A randomized controlled trial. Journal of Behavior Therapy and Experimental Psychiatry, 37, 358-371.

Wenzlaff, R. M., \& Wegner, D. M. (2000). Thought suppression. Annual Review of Psychology, 51, 59-91. 